DOI: $10.5902 / 198468627956$

\title{
Possibilidade de atuação entre saúde e educação para o desenvolvimento e aprendizagem de alunos com altas habilidades/superdotação
}

Possibility of acting between health and education for the development and learning of students with high skills / giftedness

Posibilidad de actuación entre salud y educación para el desarrollo y aprendizaje de alumnos con altas habilidades / superdotación

\section{*Edson Manoel dos Santos}

Especialização Universidade Estadual Paulista, São Paulo, São Paulo, Brasil. bioedsonm@uol.com.br

\section{** Ana Paula Pacheco Moraes Maturana}

Doutora na Universidade Federal de São Carlos, São Carlos, São Paulo, Brasil. paula.psico@hotmail.com

Recebido: 19 de julho de 2018

Aprovado: 15 de outubro de 2018

\section{RESUMO}

A Educação Especial vem ganhando cada vez mais espaço nas discussões sobre educação na realidade das escolas brasileiras. Parte integrante da categoria de alunos público alvo da educação especial, os alunos com Altas Habilidades/Superdotação (AH/SD) representam de 3 a $5 \%$ da população, mas dificilmente são identificados e suas habilidades potencializadas. Estes alunos passam despercebidos e podem apresentar problemas de comportamento e até dificuldades de aprendizagem em algumas áreas sendo encaminhados para alguns serviços, como as unidades de saúde. Sendo assim, o presente trabalho teve o objetivo de identificar possibilidades de atuação de profissionais de saúde nas estratégias de enriquecimento curricular de estudantes com indicadores de AH/SD por meio do Programa Saúde na Escola. Participaram da pesquisa 16 profissionais de saúde que responderam a um questionário sobre sua atuação nas escolas, a percepção que os profissionais têm dos alunos encaminhados pelas escolas e as possibilidades de parceria para o enriquecimento curricular dos alunos. Os profissionais de saúde desenvolvem ações de educação em saúde com foco em saúde ambiental, sexualidade, vacinação e avaliação antropométrica. No olhar dos profissionais de saúde, $75 \%$ dos alunos encaminhados para a Unidade Básica de Saúde (UBS) tinham problemas de aprendizagem e comportamentais, também $75 \%$ dos profissionais acreditam que os alunos encaminhados apresentam potencial de AH/SD. Conclui-se que a UBS pode contribuir com um olhar ampliado na 


\section{DOI: $10.5902 / 198468627956$}

avaliação dos alunos e que pode colaborar na realização de atividades de enriquecimento curricular.

Palavras-chave: Altas Habilidades; Superdotação; Programa Saúde na Escola; Enriquecimento Curricular.

\section{ABSTRACT}

Special Education has been gaining more and more space in discussions about education in the reality of Brazilian schools. An integral part of the target group of special education students, students with giftedness account for 3 to $5 \%$ of the population, but are hardly identified and their skills enhanced. These students go unnoticed and may present behavior problems and even learning difficulties in some areas being referred to the health units. Thus, the present work had the aim of identifying possibilities for health professionals to act in the curricular enrichment strategies of students with giftedness indicators through the Health in School Program. Participated in the study 16 health professionals who answered a questionnaire about their performance in schools, the perception that the professionals have of the students sent by the schools and the possibilities of partnership for the students' curriculum enrichment. Health professionals develop health education actions focused on environmental health, sexuality, vaccination and anthropometric evaluation. In the health professionals' view, $75 \%$ of the students referred to the basic health unit had learning and behavioral problems, also $75 \%$ of the professionals believe that the students referred have giftedness potential. It is concluded that basic health unit can contribute with an extended view in the evaluation of the students and that can collaborate in the accomplishment of curricular enrichment activities.

Keywords: Giftedness; Health in School Program; Curricular Enrichment.

\section{RESUMEN}

La Educación Especial viene ganando cada vez más espacio en las discusiones sobre educación en la realidad de las escuelas brasileñas. En la mayoría de los casos, los alumnos con Altas Habilidades / Superdotación (AH / SD) representan entre el 3 y el 5\% de la población, pero difícilmente se identifican y sus habilidades potencializadas. Estos alumnos pasan desapercibidos y pueden presentar problemas de comportamiento e incluso dificultades de aprendizaje en algunas áreas siendo encaminadas para algunos servicios, como las unidades de salud. Por lo tanto, el presente trabajo tuvo el objetivo de identificar posibilidades de actuación de profesionales de salud en las estrategias de enriquecimiento curricular de estudiantes con indicadores de AH / SD por medio del Programa Salud en la Escuela. En la investigación participaron 16 profesionales de salud que respondieron a un cuestionario sobre su actuación en las escuelas, la percepción que los profesionales tienen de los alumnos encaminados por las escuelas y las posibilidades de asociación para el enriquecimiento curricular de los alumnos. Los profesionales de salud desarrollan acciones de educación en salud con foco en salud ambiental, sexualidad, vacunación y evaluación antropométrica. En la mirada de los profesionales de la salud, el $75 \%$ de los alumnos 


\section{DOI: $10.5902 / 198468627956$}

encaminados a la Unidad Básica de Salud (UBS) tenían problemas de aprendizaje y comportamientos, también el $75 \%$ de los profesionales creen que los alumnos encaminados presentan potencial de AH / SD. Se concluye que la UBS puede contribuir con una mirada ampliada en la evaluación de los alumnos y que puede colaborar en la realización de actividades de enriquecimiento curricular.

Palabras clave: Altas Habilidades; La superdotación; Programa de Salud en la Escuela; Enriquecimiento Curricular.

\section{Introdução}

As políticas públicas de educação avançaram nas últimas décadas, embora ainda se tenha muito o que discutir e caminhar em relação ao acesso e a permanência dos estudantes público-alvo da educação especial (PAEE) nas escolas regulares. Em relação à matrícula desses estudantes na rede regular de ensino, segundo o Censo Escolar 2015, apontou que no ano de $2008,31 \%$ das escolas brasileiras tinham estudantes PAEE em classes regulares. Em 2015 este índice saltou para 56,6\% (BRASIL, 2016). Ainda assim, pode-se observar que o maior número de matrículas do PAEE é relacionado à alunos com deficiência intelectual, deficiência física e com transtornos globais de desenvolvimento. $O$ estudante com indicadores de Altas Habilidades/Superdotação (AH/SD), que também faz parte do PAEE e portanto tem respaldo legal tanto de matrícula e serviços, muitas vezes passa despercebido aos olhos dos professores, principalmente em escolas de periferia, onde muitos professores acreditam não haver alunos com esta característica (AZEVEDO; METTRAU, 2010).

A Política Nacional de Educação Especial na Perspectiva da Educação Inclusiva (BRASIL, 2008), define alunos com AH/SD como aqueles que demostram potencial elevado em qualquer uma das seguintes áreas, isoladas ou combinadas: intelectual, acadêmica, liderança, psicomotricidade e artes. Também apresentam elevada criatividade, grande envolvimento na aprendizagem e realização de tarefas em áreas de seu interesse.

A Política Nacional de Educação Especial na Perspectiva da Educação Inclusiva tem como objetivo assegurar a inclusão escolar de alunos com deficiência, transtornos globais de desenvolvimento e AH/SD [...] (BRASIL, 2008). Ainda, segundo esta mesma Política:

[...] na perspectiva da educação inclusiva, a educação especial passa a constituir a proposta pedagógica da escola, definindo como seu público-alvo os alunos com deficiência, transtornos globais de desenvolvimento e altas habilidades/superdotação. Nestes casos e outros, que implicam em transtornos funcionais e específicos, a educação especial atua de forma articulada com 0 ensino comum, orientando para 0 atendimento às necessidades educacionais especiais desses alunos (BRASIL, 2008 p.15). 


\section{DOI: $10.5902 / 198468627956$}

Segundo Renzulli e Reis (1997, apud RENZULLI, 2014) o comportamento de um aluno com $\mathrm{AH} / \mathrm{SD}$ consiste em comportamentos que refletem uma interação entre três grupamentos básicos de traços humanos - capacidade acima da média, elevados níveis de comprometimento com a tarefa e elevados níveis de criatividade - elementos esses que formam a famosa teoria dos três anéis de Renzulli. Os indivíduos com potencial para AH/SD são aqueles que possuem ou são capazes de desenvolver este conjunto de traços e aplicálos a qualquer área potencialmente valiosa do desempenho humano.

Para Azevedo e Mettrau (2010), em relação ao universo escolar, o estudante com AH/SD normalmente passa despercebido pela escola, seja porque é um ótimo estudante, que tira boas notas, não atrapalha a aula e não dá trabalho ao professor, ou então porque é um estudante que chama a atenção para si, interrompe a aula, tumultua. Neste caso, será identificado pela sua indisciplina e não pelo seu potencial a ser desenvolvido. Às vezes, os pais notam que seus filhos são "muito inteligentes", por terem aprendido a ler antes de outras crianças ou por apresentarem um raciocínio lógico bem desenvolvido. Há também o imaginário popular que diz que um estudante com AH/SD precisa ser um gênio, ótimo em todas as disciplinas, saber tudo e, principalmente, que este estudante não está na periferia, não está nas escolas públicas, que só será encontrado em escolas particulares e nas famílias com alto poder aquisitivo. Todas essas crenças acabam influenciando na identificação e na oferta de serviços.

Segundo o Ministério da Educação "estudos estatísticos indicam que aproximadamente 3 a $5 \%$ da população apresenta potencial acima da média estimada, em diversos contextos sociais" (BRASIL, 2006 p.19). Sendo assim, estes estudantes podem ser encontrados em todas as escolas de todo o país. Contudo, a maior dificuldade encontrada atualmente é sua identificação e o oferecimento de uma educação especializada e de qualidade (AZEVEDO; METTRAU, 2010). O trabalho de identificação precisa e deve ser realizado pela equipe escolar, por um grupo de docentes qualificados e capacitados com serviços de apoio à escola e na elaboração de parcerias (AZEVEDO; METTRAU, 2010). Por exemplo, em um trabalho de enriquecimento curricular é importante à presença de vários atores, que podem apresentar novos olhares e perspectivas aos estudantes, como por exemplo, as Unidades Básicas de Saúde (UBS) por meio do Programa Saúde na Escola (PSE).

O PSE vem contribuir para o fortalecimento de ações na perspectiva do desenvolvimento integral e proporcionar à comunidade escolar a participação em 


\section{DOI: $10.5902 / 198468627956$}

programas e projetos que articulem saúde, educação, cultura de paz e em outras redes sociais para o enfrentamento das vulnerabilidades que comprometem 0 pleno desenvolvimento de crianças, adolescentes, jovens e adultos estudantes brasileiros. Essa iniciativa reconhece e acolhe as ações de integração entre Saúde e Educação já existentes e que têm impactado positivamente na qualidade de vida dos educandos (BRASIL, 2015).

Para o PSE o trabalho de promoção da saúde com os educandos, e também com professores e funcionários, precisa ter como ponto de partida "o que eles sabem" e "o que eles podem fazer". É preciso desenvolver, em cada um, a capacidade de interpretar o cotidiano e atuar de modo a incorporar atitudes e/ou comportamentos adequados para a melhoria da qualidade de vida (BRASIL, 2015). Desse modo, profissionais de saúde podem ser bons aliados nas estratégias de enriquecimento curricular dos estudantes com indicadores de $\mathrm{AH} / \mathrm{SD}$, colaborando com a formação de cidadãos empoderados do seu papel no território que vivem promovendo saúde para toda a comunidade.

O trabalho de promoção à saúde que deve ser desenvolvido pelas equipes das UBS nas escolas parceiras, por meio do PSE pode ser uma das estratégias de enriquecimento curricular propostas aos estudantes com indicadores de AH/SD. Neste sentido, a parceria Saúde e Educação pode ser realizada em diversas perspectivas, como o conhecimento dos indicadores de saúde do território onde o estudante vive, o estudo de dados epidemiológicos sobre mortalidade, gravidez precoce, doenças prevalentes na comunidade, etc., potencializando o enriquecimento curricular proposto pela equipe pedagógica da escola.

\section{Percurso metodológico}

Para Gil (2010), a pesquisa descritiva objetiva principalmente descrever características de determinada população ou fenômeno ou estabelecer relações entre variáveis. De acordo com o autor, muitos estudos podem ser classificados sob este título e uma de suas características "está na utilização de técnicas padronizadas de coleta de dados, tais como o questionário e a observação sistemática" (GIL, 2010, p.42). Logo, a pesquisa em questão é descritiva, uma vez que é realizada um estudo, análise e interpretação de um fenômeno e valer-se de questionário para obtenção de dados.

\section{Participantes}




\section{DOI: $10.5902 / 198468627956$}

Os participantes da pesquisa foram 16 profissionais de saúde de uma UBS na cidade de São Paulo, sendo eles: 09 Agentes Comunitários de Saúde (ACS), 01 Agente de Promoção Ambiental (APA), 03 Auxiliares de Enfermagem (AE), 02 Enfermeiras e 01 Fonoaudióloga. A fim de resguardar a identidade dos participantes, estes são identificados com letras e números, sendo de P1 a P9 Agentes Comunitários de Saúde (ACS), P10 Agente de Promoção Ambiental (APA), P11 a P13 Auxiliares de Enfermagem, P14 e P15 Enfermeiras e P16 Fonoaudióloga.

Eles foram selecionados por atuarem em escolas no desenvolvimento das ações do PSE, na avaliação clínica dos alunos, avaliação antropométrica, atualização da carteira de vacinação, discussão de casos de alunos com dificuldades de aprendizagem e a realização de atividades educativas de educação em saúde para alunos, pais e professores.

\section{Instrumento}

Foi elaborado um instrumento de pesquisa com 04 perguntas abertas aplicadas aos profissionais de saúde participantes da pesquisa com o objetivo de identificar as atividades já desenvolvidas por eles nas escolas parceiras, a existência de alunos com AH/SD nas escolas e as possiblidades de atuação da UBS em propostas de enriquecimento curricular. Esse instrumento passou pela avaliação de juízes da área e aplicação de piloto.

\section{Procedimentos para a coleta e seleção de dados}

Os dados foram coletados após reuniões de rotina da UBS e aplicada ao mesmo tempo para todos os participantes. Todos receberam as mesmas instruções e responderam de forma individual a pesquisa.

\section{Procedimentos para a análise de dados}

Os dados foram analisados considerando as palavras chave em cada uma das respostas, buscando um agrupamento por temas semelhantes. Em alguns trechos foi utilizado a transcrição literal de algumas respostas de modo a ajudar a elucidar as conclusões alcançadas. A análise das respostas, seguiu a lógica do Mapa de Associação de Ideias de Mary Jane Spink (2010).

\section{Procedimentos Éticos}

É importante apontar que esta pesquisa está vinculada ao Projeto Integrado de Pesquisa "Educação Especial na Perspectiva da Educação Inclusiva: política educacional, ações escolares e formação docente", aprovada pelo Comitê de Ética em Pesquisa, da Faculdade de Filosofia e Ciências (FFC), UNESP, campus de Marília e cadastrada na 
DOI: $10.5902 / 198468627956$

Plataforma Brasil sob o n 64353216.6.0000.5406, cujo parecer é de n 1.939.831 datado de 23 de fevereiro de 2017. Vale ressaltar que esta pesquisa respeita as diretrizes e normas regulamentadoras de pesquisas envolvendo seres humanos, registradas na Resolução no 422 do CONEP.

\section{Resultados e discussão}

As respostas dos participantes foram analisadas considerando as questões norteadoras, sendo agrupadas em quatro eixos: Atividade desenvolvida pelos profissionais da UBS no PSE; Características dos alunos encaminhados pelas escolas; AH/SD nos alunos encaminhados para a UBS e Presença de alunos com AH/SD em escolas parceiras do PSE e possibilidade de atuação. Algumas respostas apresentadas na íntegra foram destacadas no formato itálico para representar melhor a opinião do participante.

\section{Encaminhamento e Atividade desenvolvida pelos profissionais da UBS no PSE}

Avaliando as respostas dadas pelos participantes da pesquisa na questão 2, as principais características dos alunos encaminhados pelas escolas para a UBS estão representados na Tabela 1.

Tabela1- Característica dos alunos encaminhados pelas escolas para a UBS

\begin{tabular}{|l|c|}
\hline Principais queixas dos alunos encaminhados para a UBS & \\
\hline Dificuldades de aprendizagem e problemas comportamentais & $75 \%$ \\
\hline Ansiedade e vulnerabilidade social & $12,5 \%$ \\
\hline Problemas familiares e violência doméstica & $12,5 \%$ \\
\hline
\end{tabular}

Fonte: Tabela elaborada pelos autores.

Exemplificando os motivos pelos quais os alunos são encaminhados para a UBS, uma participante responde que: "Geralmente são alunos com alguma queixa de aprendizagem elou problemas com o comportamento. Também são encaminhados casos que apresentam grande vulnerabilidade social' (P15).

Quadro 1. Descrição das ações desenvolvidas pelos profissionais de saúde no PSE.

\begin{tabular}{|l|l|}
\hline \multicolumn{1}{|c|}{ Profissionais } & \multicolumn{1}{c|}{ Atividades desenvolvidas } \\
\hline $\begin{array}{l}\text { Agente Comunitário de Saúde e Agente de } \\
\text { Promoção Ambiental }\end{array}$ & $\begin{array}{l}\text { Ações de educação em saúde sobre ciclo de } \\
\text { vida e manejo do mosquito Aedes aegypti e } \\
\text { os principais sintomas das doenças }\end{array}$ \\
\hline
\end{tabular}


DOI: $10.5902 / 198468627956$

\begin{tabular}{|l|l|}
\hline & $\begin{array}{l}\text { transmitidas por ele; ciclo de vida e manejo } \\
\text { de outros animais sinantrópicos como ratos, } \\
\text { baratas e pombos e sua importância para a } \\
\text { saúde. Uso racional da água e as doenças } \\
\text { de veiculação hídrica, sexualidade voltada à } \\
\text { orientação sobre a gravidez na adolescência } \\
\text { e métodos contraceptivos e rodas de } \\
\text { conversa sobre intolerância religiosa. }\end{array}$ \\
\hline Auxiliares de Enfermagem & $\begin{array}{l}\text { Avaliação Antropométrica e verificação e } \\
\text { atualização da carteira de vacinação. }\end{array}$ \\
\hline Enfermeiros e Fonoaudiólogos & $\begin{array}{l}\text { Enfermeiros realizam a avaliação } \\
\text { antropométrica e a atualização da carteira de } \\
\text { vacinação, desenvolvem rodas de conversa } \\
\text { com os alunos sobre alimentação saudável, } \\
\text { deficiência física, biopirataria, uso de drogas } \\
\text { ilícitas, bullying e intolerância religiosa. A } \\
\text { participação da fonoaudióloga foi no apoio às } \\
\text { enfermeiras em rodas de conversa com } \\
\text { professores para a discussão de casos de } \\
\text { alunos antes de serem encaminhados para } \\
\text { os serviços da rede de saúde, como por } \\
\text { exemplo, os Centros de Atenção } \\
\text { Psicossocial (CAPS) e para Ambulatórios de } \\
\text { Especialidades, conforme a necessidade. }\end{array}$ \\
\hline
\end{tabular}

Fonte: Quadro elaborado pelos autores.

As ações realizadas por Agentes Comunitários de Saúde e Agente de Promoção Ambiental estão no âmbito das ações de educação em saúde, que são atividades educativas com o objetivo de sensibilizar e orientar os alunos para temas que são pertinentes ao território do entorno da escola e que de alguma forma podem resultar em agravos à saúde, conforme preconizado no PSE. Tais dados caminham ao encontro da análise de Martins, Cristina e Delou (2016), quando apresentaram o Clube de Ciências em que os alunos foram expostos a uma grande variedade de temas, por meio de visitas, palestras, documentários, artigos, filmes e exposições. As autoras ressaltam a importância de participação em eventos e atividades extraclasse, pois a partir delas os alunos podem 


\section{DOI: $10.5902 / 198468627956$}

conhecer outros profissionais, assim como entrar em contato com o assunto de maneira prática, propiciando trocas de ideias e aprimoramento sua capacidade de argumentação e articulação (MARTINS; CRISTINA; DELOU, 2016).

Os auxiliares de enfermagem são responsáveis por identificar alunos que estejam acima ou abaixo do peso esperado ou com a carteira de vacinação desatualizada para encaminhá-los ao atendimento necessário na UBS. Os enfermeiros também realizam ações de educação em saúde e avaliação clínica dos alunos, mas direcionam suas ações nas escolas principalmente nas discussões de casos de alunos que são identificados pelos professores com problemas de comportamento ou de aprendizagem, que juntamente com a fonoaudióloga, conseguem obter maiores informações sobre os alunos, avaliar a necessidade de agendamento de atendimento na UBS ou encaminhar para outros serviços da rede de saúde para continuidade no tratamento e acompanhamento.

As atividades desenvolvidas sobre educação em saúde se enquadram no Modelo de Enriquecimento para toda a Escola (RENZULLI, 2014) e com atividades de enriquecimento do Tipo I e II (REIS; PÉREZ; FREITAS, 2015) pois podem ser aplicadas para todos os estudantes e não apenas para os já identificados superdotados. Freitas e Rech (2015), reforçam a necessidade de parceria para a realização de atividades de enriquecimento curricular, embora as autoras abordem a parceria entre o professor da sala comum e 0 professor do atendimento educacional especializado, apresenta-se aqui a UBS com um parceiro em potencial.

\section{AH/SD nos alunos encaminhados para a UBS}

$\mathrm{Na}$ questão 3, os participantes foram questionados se estes alunos encaminhados pelas escolas poderiam ter AH/SD. Para $75 \%$ dos participantes a resposta foi afirmativa, sendo estes 07 ACS e 01 APA, 02 AE e 02 enfermeiras. A participante P10 responde: "Acredito que sim, principalmente aqueles alunos com problemas de comportamento, devido períodos ociosos e achar sem interesse devido já saber a matéria", a P14 complementa: "Sim. E muitas vezes possui dificuldades de se enquadrar na metodologia utilizada na escola o que acaba demonstrando um comportamento difícil e uma deficiência na aprendizagem". Isso mostra como a parceria educação e saúde não deve se restringir a atividades pontuais, para Figueiredo, Machado e Abreu (2010), a melhor contribuição que 


\section{DOI: $10.5902 / 198468627956$}

a saúde poderia oferecer à educação reside na possibilidade de uma ação integrada e articulada, que de maneira crítica e reflexiva possa significar oportunidade de atualização dos educadores, capacitando-os para a tarefa de ministrar o discurso sobre orientação à saúde de forma transversal e interdisciplinar na escola.

Importante também é evitar que os alunos com AH/SD do tipo criativo sejam "diagnosticados" pelos professores de forma errada, quando suas habilidades para liderança, artística ou esportiva não são percebidas (MENDONÇA; MENCIA; CAPELLINI, 2015), Os que responderam Não, foram 12,5\%, sendo 01 ACS e 01 fonoaudióloga. Em sua resposta, a fonoaudióloga descreve que "em discussão de casos nunca apareceu", o que demonstra considerar apenas o olhar dos professores, já que as discussões de casos eram para os alunos identificados como "problemas" e feita com os docentes. O discurso da fonoaudióloga corrobora com o apresentado por Azevedo e Mettrau (2010), ao falar da dificuldade de identificar alunos com AH/SD, principalmente os que apresentam problemas de comportamento ou de aprendizagem. Concluindo esta questão, 01 ACS e 01 AE, o que corresponde a $12,5 \%$ do público da pesquisa, responderam que Talvez, a participante P12 ainda complementa com "em alguns poucos casos sim". O que demonstra dúvida e talvez até insegurança em sua resposta.

\section{Presença de alunos com AH/SD em escolas parceiras do PSE e Possibilidades de Atuação}

Quando questionados sobre a possibilidade de encontrar alunos com indicadores de $\mathrm{AH} / \mathrm{SD}$ nas escolas parceiras do PSE, na questão 4, 87,5\% dos participantes dizem que Sim, apenas uma ACS e a fonoaudióloga afirmaram que Não. Para ACS e APA, a colaboração que a UBS pode dar no desenvolvimento destes alunos se dá principalmente no apoio e encaminhamentos para atividades específicas. A participante P4 responde que "encaminhando para profissionais ou instituições que possam desenvolver essas habilidades", dando a entender que talvez a escola não consiga trabalhar as habilidades destes alunos, continuando a P5 responde que: "Auxiliar no encaminhamento de atividades que contribuam para o desenvolvimento dessas habilidades". A participante P10 ainda complementa que "com ações do PSE, por ser informações diferenciadas. A equipe técnica informar a equipe docente sobre as habilidades dos alunos". 


\section{DOI: $10.5902 / 198468627956$}

Com um olhar mais ampliado para a questão, a enfermeira P14 diz que "acredito que temos que modificar nosso olhar pontual para estes estudantes, utilizar uma maior sensibilidade e mostrar aos poucos para a escola que sua didática não é adequada para todos", a P15, traz um olhar mais clínico para parceria "acredito que esta colaboração possa ser feita por meio de acompanhamento em consultas (médica, enfermagem) e junto à equipe multiprofissional (psicólogo, fonoaudiólogo, psiquiatra), etc. Podendo ainda encaminhar para serviços de referência".

Os demais 12,5\% (uma ACS e uma fonoaudióloga), responderam que não é possível encontrar alunos com AH/SD nas escolas parceiras do PSE, a participante P7 fala sobre a realização de algum teste para esta identificação, enquanto P16 registra que "identificação por parte da escola e ou familiares, porém desconheço na saúde pública algum local onde seja feita a avaliação desses casos". Para Azevedo e Mettrau (2010), a identificação dos alunos com AH/SD deve ser feita por professores, colegas, pais e familiares, entre outros. Ao citar "entre outros", podemos incluir aí o profissional de saúde com o seu olhar, um olhar que não é viciado pelos vieses pedagógicos e que pode colaborar nesta tarefa da identificação destes alunos. Becker (2014), contraria os 12,5\% que afirmam não ter alunos com AH/SD nas escolas parcerias do PSE, pois para a autora, a escola é o local mais comum de encontrar alunos com tais habilidades, mas destaca também que podemos encontrar pessoas com AH/SD em qualquer área, inclusive nas instituições de longa permanência para jovens e adultos, como os reclusos do sistema penitenciário e entre os moradores em situação de rua. Para Giacomozzi et al (2012), o fortalecimento desta parceria entre educação e saúde é fundamental para abordar a questão do uso de álcool e outras drogas que acontece cada vez mais cedo entre os adolescentes, facilitando ações de prevenção e promoção à saúde que sozinha a escola não consegue conduzir.

Durante a revisão bibliográfica desta pesquisa não foram achados na literatura artigos que abordassem a questão da relação saúde-educação como uma parceira nas estratégias de enriquecimento curricular dos alunos com AH/SD, os poucos achados versavam apenas sobre a relação clínica de identificação de patologias e medicalização de alunos com alguma deficiência. $O$ único trabalho que trouxe o olhar do profissional de saúde sobre AH/SD, foi desenvolvido por Rondini, Incau e Martins (2015), que abordaram a identificação de alunos com AH/SD e alunos com Transtornos de Déficit de Atenção e Hiperatividade (TDAH) encaminhados por escolas para um Ambulatório Regional de Especialidades, ainda 


\section{DOI: $10.5902 / 198468627956$}

assim, o estudo abordou os métodos utilizados por duas médicas e uma psicóloga para concluir o diagnóstico de AH/SD ou de TDAH.

Outros estudos, como os de Martins, Pedro e Ogeda (2016) e lorio, Chaves e Anache (2016), realizaram vastas pesquisas em base de dados de teses e dissertações como a CAPES, Biblioteca Digital Brasileira de Teses e Dissertações, Plataforma Sucupira e outros Programas de Pós-Graduação com produção na área de Educação Especial. Ambas pesquisas encontraram trabalhos que abordavam a questão da identificação de alunos com $\mathrm{AH} / \mathrm{SD}$, dos instrumentos utilizados e de estratégias de enriquecimento curricular. Contudo nada foi encontrado sobre teses ou dissertações que tenha focado nas parcerias para o enriquecimento curricular de alunos, ou que abordassem o PSE ou a área da saúde como uma destas possíveis parceiras.

Outro levantamento bibliográfico sobre AH/SD como o realizado por Mendonça, Mencia e Capellini (2015), na base de dados da CAPES no período de 2000 a 2012 resultou na análise de 07 artigos sobre as estratégias de enriquecimento curricular adotadas, porém nenhuma delas relatava a participação de outros serviços ou órgãos neste processo que não fossem específicos da educação, ou seja, é inovador pensar que uma UBS possa ser parceira nas estratégias de enriquecimento curricular de alunos com AH/SD.

Para Mendonça, Mencia e Capellini (2015) o enriquecimento curricular de alunos com AH/SD pode ser feito dentro da escola e sem a necessidade de recursos em muitas ocasiões, como os laboratórios de ciências, química ou informática. Para tanto é fundamental o estabelecimento de parcerias, como as citadas pelas autoras com Universidades. No caso a presente pesquisa, apresentou como sugestão as parcerias realizadas com UBS, o que pode trazer um novo olhar para os programas de enriquecimento curricular.

\section{Considerações finais}

O trabalho permitiu verificar que os profissionais da UBS realizam diversas ações de educação em saúde com alunos e professores das EMEFs, e que os alunos são encaminhados pelas escolas com queixas de problemas de comportamento e de aprendizagem, alunos estes, que no olhar da unidade de saúde podem apresentar indicadores de AH/SD. A pesquisa também deixou evidente que a UBS pode ser um parceiro das escolas nas estratégias de enriquecimento curricular, uma vez que pode 
DOI: $10.5902 / 198468627956$

trabalhar em parceria com diferentes temáticas, considerando a multiplicidade de saberes que saúde e educação podem abordar.

A parceria entre saúde e educação deve ir muito além da prática clínica focada na medicalização, sendo o PSE um importante meio para esta aproximação saudável entre as duas áreas. No que tange a identificação de alunos com AH/SD, a escola deve ser a ordenadora deste processo, pois os profissionais da UBS não têm o olhar nem a prática pedagógica necessária para identificar um aluno com AH/SD, mas podem auxiliar neste processo, além de colaborar no desenvolvimento das atividades de enriquecimento curricular.

É necessário uma ampla avaliação dos alunos encaminhados pelas escolas para UBS com os argumentos de dificuldades de aprendizagem e problemas comportamentais, suspeitas de transtornos ou outros, pois, estes alunos podem não apresentar nenhuma patologia, além de serem talentos que a escola ainda não conseguiu identificar.

Poucos são os estudos nas áreas de $\mathrm{AH} / \mathrm{SD}$, sendo importante aprofundar novas pesquisas nestas áreas, principalmente nas relações de parcerias para o enriquecimento curricular e o PSE através das UBS pode ser um importante aliado neste processo.

\section{Referências}

AZEVEDO, S. M. L.; METTRAU, M. B. Altas Habilidades/Superdotação: Mitos e Dilemas Docentes na Indicação para o Atendimento. Psicologia, Ciência e Profissão. 2010, vol. 1, n. 30, p.32-45.

BECKER, M. A. D. É possível encontrar talentos nas ruas e instituições prisionais? Revista Educação Especial. 2014, vol. 27, n. 50, p.689-698.

BRASIL. Ministério da Educação. Secretaria de Educação Especial. Saberes e práticas da inclusão: desenvolvendo competências para 0 atendimento às necessidades educacionais especiais de alunos com altas habilidades/superdotação. 2. ed. Brasília: MEC, 2006.

Ministério da Educação. Política Nacional de Educação Especial na perspectiva da educação inclusiva. Brasília: MEC, 2008. 19 p. Documento elaborado pelo Grupo de Trabalho nomeado pela Portaria no 555/2007, prorrogada pela Portaria nำ 948/2007, entregue ao Ministro da Educação em 07 de janeiro de 2008. 


\section{DOI: $10.5902 / 198468627956$}

Ministério da Saúde. Caderno do Gestor do PSE. Brasília: Ministério da Saúde, 2015. Ministério da Educação. Censo Escolar 2015: Brasília: MEC, 2016. 19 slides, color. Disponível em:

<http://portal.mec.gov.br/index.php?option=com_docman\&view=download\&alias=36521 apresentacao-censo-escolar-divulgacao-22032016-pdf\&ltemid=30192>. Acesso em: 03 jul. 2016.

FIGUEIREDO, T. A. M.; MACHADO, V. L. T.; ABREU, M. M. S. A saúde na escola: um breve resgate histórico. Ciência e Saúde Coletiva. 2010, vol. 14, n. 2, p. 397-402.

FREITAS, S. N.; RECH, A. J. D. Atividades de enriquecimento escolar como estratégia para contribuir com a inclusão escolar de alunos com altas habilidades/superdotação. Archivos Analíticos de Políticas Educativas. 2015, vol. 23, n. 30, p. 1-19.

GIACOMOZZI, A. I. et al. Levantamento sobre uso de álcool e outras drogas e vulnerabilidades relacionadas de estudantes de escolas públicas participantes do Programa Saúde do Escolar/Saúde e Prevenção nas Escolas no município de Florianópolis. Saúde e Sociedade. 2012, vol. 21, n. 3, p. 612-622.

GIL, Antônio Carlos. Como Elaborar Projetos de Pesquisa. 5ª Ed. Editora Altlas, 2010. IORIO, N. M.; CHAVES, F. F.; ANACHE, A. A. Revisão de literatura sobre aspectos das avaliações para Altas Habilidades/Superdotação. Revista Educação Especial. 2016, vol. 29, n. 55, p. 413-428.

MARTINS, B. A.; PEDRO, K. M.; OGEDA, C. M. M. Altas habilidades/superdotação: o que dizem as pesquisas sobre estas crianças invisíveis? Psicologia Escolar e Educacional. 2016, vol. 20, n. 3, p. 561-568.

MARTINS, F. R.; CARDOSO, F. S.; DELOU, C. M. C. Clube de Ciências: atendimento a alunos com superdotação. Journal of Research in Special Educational Needs. 2016, vol. 16, n. 1, p.299-302.

MENDONÇA, L. D.; MENCIA, G. F. M.; CAPELLINI, V. L. M. F. Programas de enriquecimento escolar para alunos com altas habilidades ou superdotação: análise de publicações brasileiras. Revista Educação Especial. 2015, vol. 28, n. 53, p.721-734.

REIS, V. L.; PÉREZ, S.G. P. B.; FREITAS, S. N. Enriquecimento Extracurricular. 2014, São Paulo: AVA Moodle Unesp [Edutec]. Trata-se de texto do tipo e-book da semana 2 da disciplina Escolarização do Estudante com Altas Habilidades ou Superdotação do curso de Especialização em Educação Especial (tronco comum) Programa Rede São Paulo de Formação Docente. Acesso restrito. Disponível em: 


\section{DOI: $10.5902 / 198468627956$}

http://edutec.unesp.br/moodle/pluginfile.php/100208/mod_resource/content/15/Texto\%20 \%20AHSD\%20D07T02/index.html Acesso em: 09 dez. 2016.

RENZULLI, J. Modelo de enriquecimento para toda a escola: um plano abrangente para o desenvolvimento de talentos e superdotação. Revista Educação Especial. 2014, vol. 27, n. 50, p.539-562. Tradução: Suzana Graciela Pérez Barrera Pérez. Título original "The schoolwide enrichment model: a comprehesive plan for the development of talents end giftedness".

RONDINI, C. A.; INCAU, C; MARTINS, R. A. Concepções de profissionais de saúde sobre altas habilidades e transtorno de déficit de atenção e hiperatividade (TDAH) em crianças. Revista Educação e Cultura Contemporânea. 2015, vol. 13, n. 32, p. 152-170.

SPINK, M. J. Linguagem e produção de sentidos no cotidiano. Rio de Janeiro: Centro Edelstein de Pesquisas Sociais. 2010.

\section{Correspondência}

Edson Manoel dos Santos - Universidade Estadual Paulista Júlio de Mesquita Filho, R. Pamplona, 145 - Cerqueira César, CEP: 01405-000, São Paulo, São Paulo, Brasil.

Th is work is licensed under a Creative Commons Attribution-NonCommercial 4.0 International (CC BY-NC 4.0) 